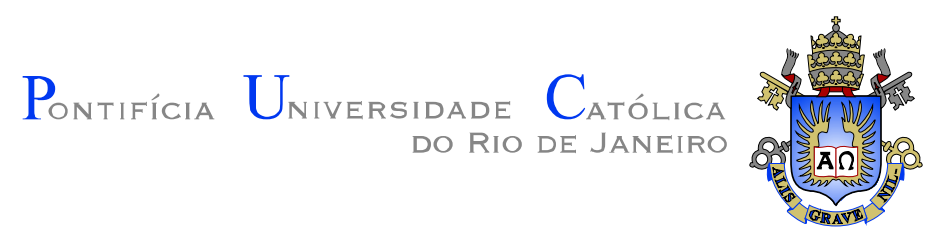

Carlos Vinicio Rodríguez Ron

Caracterização do Canal Rádio em Banda Larga na Faixa de 3,5 GHz em Ambiente Urbano

Tese de Doutorado

Tese apresentada ao Programa de Pós-graduação em Engenharia Elétrica do Departamento de Engenharia Elétrica da PUC-Rio como parte dos requisitos parciais para obtenção do título de Doutor em Engenharia Elétrica

Orientador: Prof. Dr. Luiz Alencar Reis da Silva Mello 


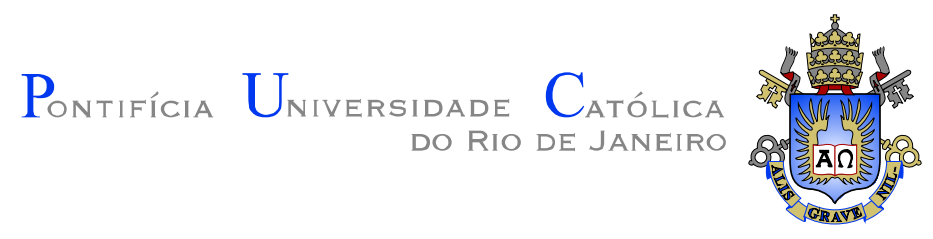

Carlos Vinicio Rodríguez Ron

\title{
Caracterização do Canal Rádio em Banda Larga na Faixa de 3,5 GHz em Ambiente Urbano
}

Tese apresentada como requisito parcial para obtenção do grau de Doutor pelo Programa de Pós-Graduação em Engenharia Elétrica do Departamento de Engenharia Elétrica do Centro Técnico Científico da PUC-Rio.

Aprovada pela Comissão Examinadora abaixo assinada.

\author{
Dr. Luiz Alencar Reis da Silva Mello \\ Orientador \\ Centro de Estudos em Telecomunicações / PUC-Rio \\ Dr. Adaildo Gomes D'Assunção \\ UFRN \\ Dr. Gervásio Protásio dos Santos Cavalcante \\ UFPA \\ Dr. Cássio Gonçalves do Rego \\ UFMG
}

Dr. Flávio José Vieira Hasselmann

Centro de Estudos em Telecomunicações/PUC-Rio

Dr. Gláucio Lima Siqueira

Centro de Estudos em Telecomunicações/PUC-Rio

Dr. Rodolfo Sabóia Lima de Souza

Inmetro

Prof. José Eugenio Leal

Coordenador Setorial do Centro

Técnico Cintífico - PUC-Rio

Rio de Janeiro, 04 de Setembro de 2009 
Todos os direitos reservados. É proibida a reprodução total ou parcial do trabalho sem autorização da universidade, do autor e do orientador.

\section{Carlos Vinicio Rodríguez Ron}

Graduou-se em Engenharia Eletrônica com ênfase em Telecomunicações na Escola Politécnica do Exército em Quito Equador em 2000. Obteve o título de Mestre em Ciências em Engenharia Elétrica pelo Instituto Militar de Engenharia em 2003 na área de Sistemas de Comunicações. Trabalhou junto com a empresa Otecel S.A (BellSouth) do Equador em sistemas de telecomunicações sem fio em engenharia de Rádio-Frequência, tráfego e comutação, posteriormente integrou o quadro de engenheiros da Empresa WiNGS Telecom Ltda.

Ficha Catalográfica

Rodríguez, Carlos

Caracterização do Canal Rádio em Banda Larga na Faixa de 3,5 GHz em Ambiente Urbano / Carlos Vinicio Rodríguez Ron; orientador: Dr. Luiz Alencar Reis da Silva Mello. - Rio de Janeiro: PUC-Rio, Departamento de Engenharia Elétrica, 2009.

v., $161 \mathrm{f:}$ il. ; $29,7 \mathrm{~cm}$

1. Tese (doutorado) - Pontifícia Universidade Católica do Rio de Janeiro, Departamento de Engenharia Elétrica.

Inclui referências bibliográficas.

1. Engenharia Elétrica - Tese. 2. Caracterização de canal banda larga;. 3. Ambientes Urbanos;. I. Silva Mello, Luiz. II. Pontifícia Universidade Católica do Rio de Janeiro. Departamento de Engenharia Elétrica. III. Título. 


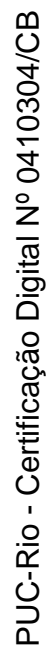

A Alessandra, João Carlos, meus pais Carlos Rosendo e Isabel de Lourdes, e, irmãs Maria de Lourdes e Maria Fernanda Rodríguez Ron 


\section{Agradecimentos}

A Deus pela força e pelo que tem me mostrado ao longo destes anos.

Ao meu orientador Professor Luiz Alencar Reis da Silva Mello pelo grande apoio, na orientação do doutorado e pela amizade.

À Professora Marlene Sabino Pontes pela confiança, apoio profissional e conselhos profissionais e pessoais que me ajudaram muito nestes anos.

À minha esposa Alessandra pelo amor, paciência e pelo entusiasmo que sempre participa junto comigo a cada desafio que se apresenta pela frente.

Ao meu filho João Carlos que a cada dia me ensina muito e com a sua alegria me transmite esperança e futuro.

Aos meus pais que sempre me apoiaram de forma incondicional, pela educação e os maravilhosos momentos de família que mesmo com a separação pela distância, ainda temos.

Às minhas irmãs que sempre com a sua graça, simpatia e amizade infinita me ajudaram em todos os aspectos.

Ao DITEL (Divisão de Telecomunicaçãoes) do INMETRO (Instituto Nacional de Metrologia) pelas facilidades laboratoriais e de instrumentação colocadas à disposição do trabalho de Tese.

Aos membros do DITEL, Dr. Rodolfo Sabóia Lima de Souza, Dr. Claiton Pereira Colvero e Dr. Pedro Vladimir Gonzáles pelo suporte nas etapas de especificação, testes e medições.

Ao CNPq e à PUC-Rio, pelos auxílios concedidos, sem os quais este trabalho não poderia ter sido realizado.

Aos meus colegas do CETUC da PUC-Rio pela troca de idéias e ajuda no desenvolvimento da parte experimental do trabalho. 


\section{Resumo}

Rodríguez, Carlos; Silva Mello, Luiz(Orientador). Caracterização do Canal Rádio em Banda Larga na Faixa de $3,5 \mathrm{GHz}$ em Ambiente Urbano. Rio de Janeiro, 2009. 161p. Tese de Doutorado - Departamento de Engenharia Elétrica, Pontifícia Universidade Católica do Rio de Janeiro.

O correto planejamento de novas redes de comunicação sem fio de voz e dados representa um grande desafio ao incluir serviços cada vez mais avançados com diferentes requisitos de qualidade, suporte a mobilidade, altas taxas de transmissão e capacidades de tráfego elevadas. Os ambientes nos quais estas redes operam são dos mais diversos e contemplam desde áreas abertas até centros urbanos com alta densidade de edificações.

O presente trabalho apresenta resultados experimentais que permitem caracterizar o canal de rádio-propagação num ambiente urbano com mobilidade do receptor. Como resultado de campanhas de medições, são identificados modelos do canal que permitem ao projetista da rede definir a melhor configuração de parâmetros e critérios de projeto para a implantação de uma rede móvel de acesso sem fio em banda larga. As medições foram realizadas nos bairros da Gávea, Leblon e Lagoa, no Rio de Janeiro, com estação transmissora instalada na PUC-Rio (prédio LEME) na frequência de $3,5 \mathrm{GHz}$, destinada para novos serviços móveis.

Os principais resultados obtidos são: o desenvolvimento de uma metodologia de medição simples e precisa, utilizando a técnica OFDM, e das ferramentas de softuare para pré-processamento e análise de dados; o desenvolvimento de modelos semi-empíricos para a previsão da perda média de propagação; a obtenção de perfis de retardo do canal e dos valores médio e desvio padrão do retardo em condições de visibilidade (LOS) e obstrução (NLOS), e de expressões empíricas para sua estimativa; a obtenção do valor médio das amplitudes relativas dos multipercursos e sua modelagem em função do valor de retardo relativo.

\section{Palavras-chave}

Caracterização de canal banda larga; Ambientes Urbanos; 


\section{Abstract}

Rodríguez, Carlos; Silva Mello, Luiz(Advisor). Wideband Radio Channel Characteristics at $3,5 \mathrm{GHz}$ in Urban Regions. Rio de Janeiro, 2009. 161p. PhD Thesis - Departamento de Engenharia Elétrica, Pontifícia Universidade Católica do Rio de Janeiro.

Best practices of radio planning for new wireless networks with mixed traffic of voice and data are a big technical challenge due to the need to provide of advanced services with different quality requirements, high data throughput, mobility and high traffic demands. Environments for operation of this kind of networks are diverse and include open areas with low population density as well as urban centers.

This thesis presents experimental results obtained to characterize the wideband radiopropagation channel on urban environments with a mobile receiver. The analysis of measured data allows the identification of channel models and propagation mechanisms needed for the design engineer to define the best parameter configuration and project criteria in the deployment of a wideband mobile wireless network. The measurement campaign was executed on Gavea neigborhood in Rio de Janeiro city with a transmitter station located in a PUC-Rio university building (Leme Building) operating at $3,5 \mathrm{GHz}$, frequency used for the new wideband mobile services.

The main results are: the development of a simple and precise methodology for measuring, using the OFDM technique OFDM, and the software tools for pre-processing and analysis; the development of semi-empirical models for predicting the average propagation loss in the urban environment; measurements of multipath delay profiles of the channel and the average values and standard deviation of the RMS delay spread in visibility (LOS) and obstruction (NLOS) conditions, and derivation of empirical expressions for their estimation; measurements of the average values of multipath amplitudes its modeling in function of the relative delay.

\section{Keywords}

Wideband Channel Charcterisation; Urban Environments; 


\section{Sumário}

$\begin{array}{lr}\text { Sumário das abreviaturas } & 14\end{array}$

$\begin{array}{ll}\text { Sumário das notações } & \mathbf{1 5}\end{array}$

1 Introdução $\quad 17$

1.1 Um pouco da História 18

$\begin{array}{lll}1.2 & \text { Objetivos da tese } & 23\end{array}$

$\begin{array}{lll}1.3 & \text { Proposta do trabalho } & 24\end{array}$

2 O Canal de Propagação Rádio Móvel $\quad \mathbf{2 6}$

2.1 Modelamento do Canal em Banda Estreita 27

2.2 Caracterização de Canais em Banda Larga 39

3 Técnicas de medição $\quad \mathbf{4 8}$

3.1 Sondagem por pulso 48

3.2 Técnica no Domínio do Tempo - Pulse Compression 49

3.3 Técnica no Domínio da Frequência 50

$4 \quad$ Set- Up de medições $\quad 61$

4.1 Área das medições $\quad 61$

4.2 Set-up de transmissão $\quad 63$

4.3 Set-up de recepção

4.4 Obtenção do sinal de calibração $\operatorname{CAL}(\mathrm{f}) \quad 76$

5 Campanha de medição $\quad 81$

5.1 Avaliação da situação espectral $\quad 81$

5.2 Banco de dados de medição 84

5.3 Medições de cobertura 84

5.4 Medições do Canal Banda Larga 89

6 Análise de Resultados $\quad 92$

6.1 Mapas utilizados para as análises $\quad 92$

6.2 Comparação com modelos de predição de perda de propagação ITU-R 93

6.3 Medições vs. Rec. ITU-R P.1411 94

6.4 Medições vs. Rec. ITU-R P.1546 95

6.5 Medições vs. Modelo SUI 97

6.6 Modelos propostos 99

6.7 Caracterização de Canal Banda Larga 107

7 Conclusões e Recomendações 118

$\begin{array}{ll}\text { Referências Bibliográficas } & 121\end{array}$

$\begin{array}{ll}\text { A Código utilizado no Matlab } & 127\end{array}$ 
B Especificação de equipamentos $\quad 145$

C Autorização de Uso Temporário de Radiofrequências 150

D Banco de Dados $\quad 151$

E Rotas de medição 153 


\section{Lista de figuras}

1.1 Evolução de sistemas sem fio - Visão ITU [Fonte: ITU]. 22

1.2 Implementações de redes CDMA (cdmaOne, CDMA2000 1X e 1xEV-DO) [Fonte: CDG].

2.1 Comportamento de sinal em ambiente urbano. 27

2.2 Componentes no plano vertical. 28

2.3 Componentes no plano horizontal. 28

2.4 Banco vetorial de ambiente de simulação, bairro Gávea - Rio de Janeiro

2.5 Relação entre funções - Determinísticas.

2.6 Decaimento exponencial e Perfil de retardos.

3.1 Sistema de Transmissao por Blocos. 52

3.2 Sinal OFDM - Domínio da Frequência. $\quad 57$

3.3 Sinal OFDM - Domínio do Tempo. 58

3.4 Perfil de Retardos - NLOS - CFAR. 60

4.1 Azimute 90 graus NM. 61

4.2 Azimute 150 graus NM. 62

4.3 Azimute 30 graus NM. 62

4.4 Localização da antena do transmissor. 63

4.5 Perfil de terreno - Determinação de inclinação da antena. 63

$\begin{array}{lll}4.6 & \text { OFDM - FFT } 20 \mathrm{MHz} . & 65\end{array}$

4.7 OFDM - FFT $7 \mathrm{MHz}$.

$\begin{array}{lll}4.8 & \text { OFDM - FFT } 3,5 \mathrm{MHz} . & 66\end{array}$

$\begin{array}{lll}4.9 & \text { OFDM - FFT } 1,75 \mathrm{MHz} . & 67\end{array}$

$\begin{array}{ll}4.10 \text { OFDM - } 20 \mathrm{MHz} . & 67\end{array}$

4.11 OFDM - $7 \mathrm{MHz}$. 68

4.12 OFDM - 3,5 MHz. 68

$\begin{array}{ll}4.13 \text { OFDM - 1,75 MHz. } & 69\end{array}$

4.14 Linearidade do amplificador. $\quad 70$

4.15 Instalação de equipamentos. $\quad 70$

$\begin{array}{ll}4.16 \text { Transmissor. } & 71\end{array}$

4.17 Características Elétricas Cabo LMR-400. 73

$\begin{array}{ll}4.18 \text { OFDM - } 20 \mathrm{MHz} . & 74\end{array}$

4.19 OFDM - $20 \mathrm{MHz}$.

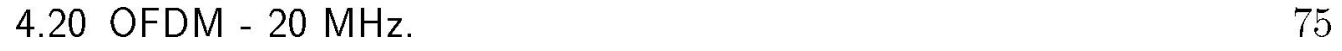

4.21 Ambiente de medição para calibração. $\quad 76$

4.22 Sinal para calibração $1,75 \mathrm{MHz}$.

4.23 Sinal para calibração 3,5 MHz.

$\begin{array}{ll}4.24 \text { Sinal para calibração } 7 \mathrm{MHz} \text {. } & 78\end{array}$

4.25 Sinal para calibração $20 \mathrm{MHz}$. 78

$\begin{array}{ll}4.26 \text { Resposta de equipamentos para } 1,75 \mathrm{MHz} \text {. } & 79\end{array}$

4.27 Resposta de equipamentos para 3,5 $\mathrm{MHz}$. $\quad 79$

4.28 Resposta de equipamentos para $7 \mathrm{MHz}$. 80 
5.1 Consulta SITAR - Sistemas ponto-a-ponto e ponto-área. 82

5.2 Rota realizada para varredura de espectro. 82

5.3 Varredura de espectro. 83

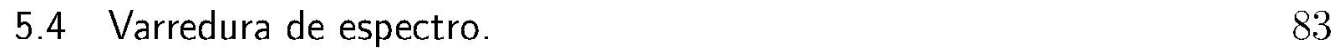

5.5 Rota realizada para medição. 85

5.6 Valores de potência recebida. 86

5.7 Valores de potência recebida Gávea. 86

5.8 Perda de propagação vs distância para distâncias menores a 1 km - LOS.

5.9 Perda de propagação vs distância para distâncias menores a $1 \mathrm{~km}$ - NLOS.

5.10 Perda de propagação vs distância para distâncias maiores a $1 \mathrm{~km}$ LOS.

5.11 Perda de propagação vs distância para distâncias maiores a 1 km NLOS.

5.12 Mapa de grupo de medições 02/11/2008 para $20 \mathrm{MHz}$.

5.13 Perfil de retardos em função das amostras 02/11/2008 part 1 para $20 \mathrm{MHz}$.

5.14 Perfil de retardos em função da distância entre transmissor e receptor $02 / 11 / 2008$ part 1 para $20 \mathrm{MHz}$.

5.15 Perfil de retardos em função das amostras entre transmissor e receptor 02/11/2008 part 1 para $20 \mathrm{MHz}$ - CFAR.

5.16 Perfil de retardos em função da distância entre transmissor e receptor 02/11/2008 part 1 para $20 \mathrm{MHz}$ - CFAR.

6.1 Mapa do bairro Gávea e prédios.

6.2 Perda de propagação vs distância, medição e ITU R P.1411 para LOS.

6.3 Perda de propagação vs distância, medição e ITU R P.1411 para NLOS.

6.4 Lrts - Rec. ITU-R P.1411-3.

6.5 Perda de propagação vs distância, medição e ITU R P.1546 para LOS.

6.6 Perda de propagação vs distância, medição e ITU R P.1546 para NLOS.

6.7 Perda de propagação vs distância, medição e SUI Model.

6.8 Perda de propagação vs distância, Modelo 0 - Primeiro ajuste.

6.9 Perda de propagação vs distância, Modelo 1.

6.10 Distribuição estatística de erros, Modelo 1 - LOS.

6.11 Distribuição estatística normal e resíduos, Modelo 1 - LOS. 101

6.12 Valores medidos vs predição, Modelo 1 - LOS. 101

6.13 Residuos vs Predição, Modelo 1 - LOS. 102

6.14 Distribuição estatística de erros, Modelo 1 - NLOS. 102

6.15 Distribuição estatística normal e resíduos, Modelo 1 - NLOS. 103

6.16 Valores medidos vs predição, Modelo 1 - NLOS. 103

6.17 Perda de propagação vs distância, Modelo 2. 104

6.18 Distribuição estatística de erros, Modelo 2. 105 
6.19 Distribuição estatística normal e resíduos, Modelo 2.

6.20 Valores medidos vs predição, Modelo 2. 106

6.21 Resíduos vs Predição, Modelo 2. 106

6.22 Retardo RMS em função da distância. 109

6.23 Retardo médio em função da distância. 110

6.24 Modelo para valor médio do retardo RMS em função da distância (0 a 700 metros) LOS.

6.25 Modelo para valor médio do retardo RMS em função da distância (700 a 1.500 metros) LOS.

6.26 Modelo para valor médio do retardo RMS em função da distância (maior a 1500 metros) LOS.

6.27 Modelo para desvio padrão do retardo RMS em função da distância (0 a 700 metros) LOS.

6.28 Modelo para desvio padrão do retardo RMS em função da distância (700 a 1.500 metros) LOS.

6.29 Modelo para desvio padrão do retardo RMS em função da distância (maior a 1.500 metros) LOS.

6.30 Perfil de retardos $20 \mathrm{MHz}$ - LOS.

6.31 Perfil de retardos $20 \mathrm{MHz}$ - NLOS.

6.32 Perfil de retardos $20 \mathrm{MHz}$ Situações LOS e NLOS.

6.33 Perfil de retardos 1,75 MHz Situações LOS e NLOS.

6.34 Perfil de retardos 3,5 MHz Situações LOS e NLOS.

6.35 Perfil de retardos $7 \mathrm{MHz}$ Situações LOS e NLOS.

6.36 Modelo de coeficiente constante em função da largura de canal. 


\section{Lista de tabelas}

1.1 Telefonia Móvel 1G - Cronologia. 21

2.1 Tipos de Terreno e parâmetros - Modelo SUI. 31

3.1 Equipamentos de medição - Técnicas de Sondagem. 49

4.1 Parâmetros de medição. $\quad 65$

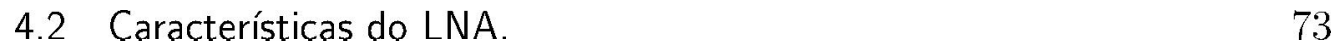

4.3 Consumo de energia na viatura. 76

6.1 Erros de medição e cálculo para modelo SUI. 98

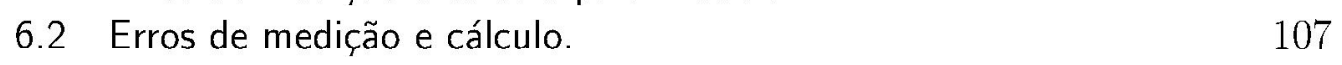

6.3 Erros de medição e cálculo - Perfil de retardos. 108

D.1 Formato de arquivos de captura MS2781 - Análise Espectral 151

D.2 Formato de arquivos de captura MS2781 - Campanha de medições 152 


\section{Sumário das abreviaturas}

$\begin{array}{ll}\text { 1G } & \text { Primeira Geração de telefonia celular } \\ \text { 2G } & \text { Segunda Geração de telefonia celular } \\ \text { 3G } & \text { Terceira Geração de telefonia celular } \\ \text { 4G } & \text { Quarta Geração de telefonia celular } \\ \text { BER } & \text { Bit Frror Rate } \\ \text { CDMA } & \text { Code Division Multiple Access } \\ \text { CP } & \text { Cyclic Prefix, prefixo ciclico } \\ \text { FDMA } & \text { Frequency Division Multiple Access } \\ \text { GSM } & \text { Global Systems for Mobile Telecommunications } \\ \text { IBI } & \text { Interblock Interference } \\ \text { INMETRO } & \text { Instituto Nacional de Metrologia } \\ \text { IMT-2000 } & \text { International Mobile Telecommunications-2000 } \\ \text { ISI } & \text { Inter Symbol Interference } \\ \text { LTE } & \text { Iong Term Fvolution } \\ & \text { Zeros } \\ \text { OFDM } & \text { Orthogonal Frequency-Division Multiplexing } \\ \text { OFDMA } & \text { Orthogonal Frequency-Division Multiple Access } \\ \text { SUI } & \text { Stanaford University Interim } \\ \text { UMTS } & \text { Iniversal Mobile Telecommunications System }\end{array}$




\section{Sumário das notações}

$\begin{array}{ll}x & \text { Escalar } \\ \boldsymbol{x} & \text { Vetor } \\ \boldsymbol{X} & \text { Matriz } \\ (\cdot)^{T} & \text { Transposto } \\ (\cdot)^{H} & \text { Hermitiano } \\ (\cdot)^{*} & \text { Complexo conjugado } \\ \boldsymbol{I}_{N} & \text { Matriz identidade de tamanho } N \\ \mathbb{E}[\cdot] & \text { Valor esperado } \\ \Re(\cdot) & \text { Parte real de um número complexo } \\ \Im(\cdot) & \text { Parte imaginária de um número complexo }\end{array}$




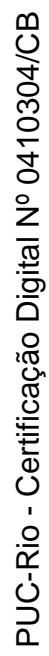

"quando Deus quer uma obra, os obstáculos tornam-se meios."

Padre Júlio Chevalier, . 